\title{
Malformações, abortos e mortalidade embrionária em ovinos causada pela ingestão de Mimosa tenuiflora (Leguminosae) ${ }^{1}$
}

\author{
José Rômulo Soares dos Santos² ${ }^{2}$ Antônio F.M. Dantas ${ }^{2}$ e Franklin Riet-Correa ${ }^{2 *}$
}

\begin{abstract}
Santos J.R.S., Dantas A.F.M. \& Riet-Correa F. 2012. [Malformations, abortion, and embryonic death in sheep caused by the ingestion of Mimosa tenuiflora (Leguminosae).] Malformações, abortos e mortalidade embrionária em ovinos causada pela ingestão de Mimosa tenuiflora (Leguminosae). Pesquisa Veterinária Brasileira 32(11):1103-1106. Hospital Veterinário, CSTR, Universidade Federal de Campina Grande, Campus de Patos, 58708-110 Patos, PB, Brazil. E-mail: franklin.riet@pq.cnpq.br

To determine the teratogenicity of Mimosa tenuiflora, 15 sheep, divided into two groups, were introduced into an area invaded by the plant. Group 1 consisted of six pregnant ewes that were introduced into the experimental area 20 days after mating. Group 2 consisted of nine non pregnant sheep and a ram introduced into the area at the start of the experiment. The experiment began in October 2009, during the dry season, when M. tenuiflora was seeding. During this period the M. tenuiflora plants were pruned to $40 \mathrm{~cm}$ tall and the branches with leaves and seeds were provided to the experimental sheep in the same area. After prunning $M$. tenuiflora began to sprout even in the dry season, and the sheep were free to graze the plant. They also received concentrate ration in amount equivalent to $1 \%$ of their body weight. After the first rains in mid-January 2010, when the herbaceous layer appeared, the pregnant sheep were confined in stalls, and M. tenuiflora was cut in the field and given to the sheep until the end of the experiment. Every 15 days each sheep was examined by ultrasound to control pregnancy. In Group 1, three sheep aborted single fetuses without malformations. One sheep delivered two lambs, one with hyperflexion of the proximal inter-phalangeal joint of the right forelimb and another without malformations. Another sheep delivered a lamb with a hyperflexion of both hindlimbs in the region of the tarsal-metatarsal joint. Only one sheep delivered a normal lamb. In Group 2, one sheep aborted a fetus without malformations and five delivered normal lambs. Three sheep of this group returned to estrus repeatedly and did not get pregnant during the mating period, suggesting embryonic loss. Thirty two ewes and one ram, that stayed in a paddock neighbor to the experimental paddock and were used as control group, delivered normal lambs. It is concluded that $M$. tenuiflora cause malformations, embryonic mortality and abortion in sheep.
\end{abstract}

INDEX TERMS: Mimosa tenuiflora, abortion, malformations, embryonic death, plant poisoning.

RESUMO.- Para determinar a teratogenicidade de Mimosa tenuiflora, 15 ovelhas, distribuídas em dois grupos, foram introduzidos em área invadida pela planta. 0 Grupo 1 , com seis ovelhas prenhes, foi introduzido na área experimental 20 dias após o acasalamento. 0 Grupo 2, formado por nove ovelhas não prenhes e um carneiro, foi introduzido na área experimental no início do experimento. 0 experimento começou no mês de outubro de 2009, período de estiagem,

\footnotetext{
${ }^{1}$ Recebido em 9 de março de 2012.

Aceito para publicação em 4 de agosto de 2012.

${ }^{2}$ Hospital Veterinário, Centro de Saúde e Tecnologia Rural (CSTR), Universidade Federal de Campina Grande (UFCG), Campus de Patos, 58708-110 Patos, PB, Brasil. *Autor para correspondência: franklin.riet@pq.cnpq.br
}

quando M. tenuiflora estava sementando. Nesse período as plantas foram rebaixadas a $40 \mathrm{~cm}$ de altura e os galhos com folhas e sementes foram disponibilizados para os ovinos na mesma área onde $M$. tenuiflora foi rebaixada. $M$. tenuiflora começou a rebrotar ainda na estação seca antes do período de chuvas. No período de rebrota, as ovelhas ficavam livres para pastar M. tenuiflora e recebiam concentrado em quantidade equivalente a $1 \%$ do peso vivo. Após as primeiras chuvas, em meados de janeiro do ano seguinte, quando o estrato herbáceo apareceu, essas ovelhas foram confinadas em baias, onde $M$. tenuiflora foi fornecida até o fim do experimento. A cada 15 dias eram realizados exames ultrassonográficos para acompanhamento da gestação. No Grupo 
1 , três ovelhas abortaram, cada uma um feto sem malformações. Outra ovelha pariu dois cordeiros, um com hiperflexão na articulação inter-falangeana proximal no membro torácico direito e outro sem malformações. Outra ovelha pariu um cordeiro com hiperflexão dos dois membros pélvicos na região da articulação tarso-metatársica. No grupo formado pelas ovelhas que foram acasaladas na área experimental, uma ovelha abortou um feto sem malformações e cinco pariram cordeiros normais. Três das ovelhas desse grupo não emprenharam durante todo o período experimental, mostrando retornos repetidos ao cio, sugerindo perda embrionária. Trinta e duas ovelhas e um carneiro, que permaneceram numa área vizinha a área experimental e foram utilizados como controle, pariram cordeiros normais. Conclui-se que $M$. tenuiflora, além de causar malformações causa, também, mortalidade embrionária e abortos em ovelhas.

TERMOS DE INDEXAÇÃO: Mimosa tenuiflora, aborto, malformações, mortalidade embrionária, plantas tóxicas.

\section{INTRODUÇÃO}

Diversas plantas, incluindo Astragalus lentiginosus, Conium maculatum, Lupinus spp, Nicotiana glauca, Nicotiana tabacum, Oxytropis spp. e Veratrum californicum, causam anomalias craniofaciais e malformações do sistema músculo-esquelético em ruminantes, como artrogripose, braquignatia, ciclopia e palatosquise (Keeler 1990, Cheeke 1998, Gardner et al. 1998, Panter et al. 2000, 2011, James et al. 2004). Os principais teratógenos descritos em plantas são alcaloides indolizidínicos, piperidínicos e quinolizidinicos (Keeler 1984, Panter et al. 1994).

No nordeste do Brasil, a ingestão de Mimosa tenuiflora (jurema preta) tem sido associada a malformações congênitas, incluindo anomalias ósseas craniofaciais, malformações oculares e artrogripose em ovinos, caprinos e bovinos (Medeiros et al. 2005, Nóbrega Júnior et al. 2005, Dantas et al. 2010). Pimentel et al. (2007) testaram os efeitos do consumo de $M$. tenuiflora durante todo o período gestacional em cabras gestantes e verificaram malformações, semelhantes às observadas nos casos espontâneos, em $75 \%$ das crias. Medeiros et al. (2008) demonstraram o efeito teratogênico de $M$. tenuiflora ao reproduzir malformações (fenda palatina, escoliose, esterno bífido, aplasia de esternebras, hipoplasia do osso nasal) em $84 \%$ dos filhotes de ratas que receberam ração contendo $10 \%$ de sementes, entre o 6으 e $21^{\circ}$ dia de gestação.

Casos espontâneos de anomalias congênitas são descritos em ovinos criados extensivamente no semiárido associados ao consumo de M. tenuiflora (Dantas et al. 2010); no entanto, a ocorrência de defeitos congênitos em ovinos relacionada ao consumo dessa planta não foi, ainda, confirmada experimentalmente. Assim, o objetivo desse trabalho foi reproduzir malformações fetais em ovelhas prenhes que ingeriram M. tenuiflora durante a gestação.

\section{MATERIAL E MÉTODOS}

O experimento foi realizado nas dependências do Hospital Veterinário, da Universidade Federal de Campina Grande, situado no município de Patos e também na Fazenda Caicú, município de São José de Espinharas, Paraíba. Na fazenda foi demarcada uma área, de três hectares (área experimental), 80\% invadida por Mimosa tenuiflora. Nos outros $20 \%$ havia Croton sonderianus (marmeleiro) e Caesalpinia bracteosa (catingueira). A área está situada na longitude $\mathrm{S} 37^{\circ} 29^{\prime} 17^{\prime \prime}$, latitude $\mathrm{W} 6^{\circ} 45^{\prime} 21^{\prime \prime}$ com altitude de $228 \mathrm{~m}$.

Foram utilizadas 15 ovelhas e dois carneiros da raça Santa Inês, todos em idade reprodutiva. Inicialmente, as ovelhas foram submetidas a exame ultrassonográfico para confirmar diagnóstico negativo de gestação e os machos foram submetidos a exames andrológicos a fim de determinar sua capacidade reprodutiva. Posteriormente, as ovelhas foram identificadas e vermifugadas. As 15 ovelhas foram distribuídas aleatoriamente em dois grupos. As seis ovelhas do Grupo 1 ficaram em curral com reprodutor no Hospital Veterinário. 0 reprodutor era marcado com tinta vermelha na região do tórax e abdômen para detecção do dia de cobertura pela impregnação da tinta no dorso das ovelhas. Vinte dias após a cobertura era realizado exame ultrassonográfico; se confirmada a gestação, a ovelha era encaminhada à área experimental. 0 Grupo 2 era composto por 9 ovelhas que ficaram na área experimental com outro carneiro, também marcado com tinta vermelha. No final do dia, as ovelhas eram recolhidas a baia determinando-se a data de cobertura caso as ovelhas apresentassem mancha vermelha no dorso. Após 20 dias do dia de cobertura procedia-se a ultrassonografia.

0 experimento foi iniciado no mês de outubro de 2009, período seco, quando $M$. tenuiflora estava sementando e as ovelhas consumiam as sementes que estavam no chão. Nesse período as plantas foram rebaixadas a $40 \mathrm{~cm}$ de altura para permitir a rebrota e os galhos com sementes e folhas remanescentes foram disponibilizados na mesma área a campo, para o acesso das ovelhas, tempo em que se aguardava a rebrota. $M$. tenuiflora começou a rebrotar no mês de novembro, ainda na estação seca. No período de rebrota, as ovelhas ficavam livres para pastar jurema preta que era a única fonte de alimentação volumosa, e recebiam $1 \%$ de concentrado com base no peso vivo. Após as primeiras chuvas, em meados de janeiro, brotou o estrato herbáceo, composto principalmente por Aristida setifolia (capim panasco), Brachiaria plantaginea (milhã), Senna obtusifolia (mata-pasto) e Sida cordifolia (malva branca). A partir daí, as ovelhas foram confinadas em baias e M. tenuiflora foi fornecida até o fim do experimento. Os galhos da planta eram suspensos nas paredes laterais das baias para facilitar a sua ingestão. A alimentação durante todo o experimento era constituída de jurema preta à vontade e ração comercial em quantidade equivalente a $1 \%$ do peso corporal e água ad libitum. A cada 15 dias era realizada ultrassonografia para constatação da prenhez. Durante todo o experimento os animais foram observados diariamente para detectar a ocorrência de abortos. As ovelhas com os cordeiros que nasceram vivos malformados foram mantidas sob observação no Hospital Veterinário.

Durante o experimento, 32 ovelhas e um reprodutor permaneceram numa área de 17 hectares, da mesma propriedade, vizinha à área experimental, na qual havia escassas plantas de $M$. tenuiflora. Esse rebanho foi, também, acompanhado, servindo como grupo controle.

\section{RESULTADOS}

No Grupo 1, das seis ovelhas que foram introduzidas na área experimental no $20^{\circ}$ dia de gestação, três abortaram fetos sem malformações, aos 52, 58 e 146 dias de gestação, respectivamente. Nas necropsias não foram encontradas sinais de viabilidade nem lesões significativas. Outra ovelha desse grupo pariu dois cordeiros, sendo que um apre- 
sentava hiperflexão na articulação inter-falangeana proximal no membro torácico direito (Fig.1); esta malformação reverteu-se após duas semanas. Outra ovelha pariu um cordeiro com hiperflexão dos membros pélvicos na região da articulação tarso-metatársica (Fig.2). Esta lesão regrediu em 28 dias. Apenas uma ovelha desse grupo teve parto normal e pariu um cordeiro sem malformações.

No Grupo 2, das nove ovelhas, que estavam na área experimental, seis emprenharam durante o período seco (ou-

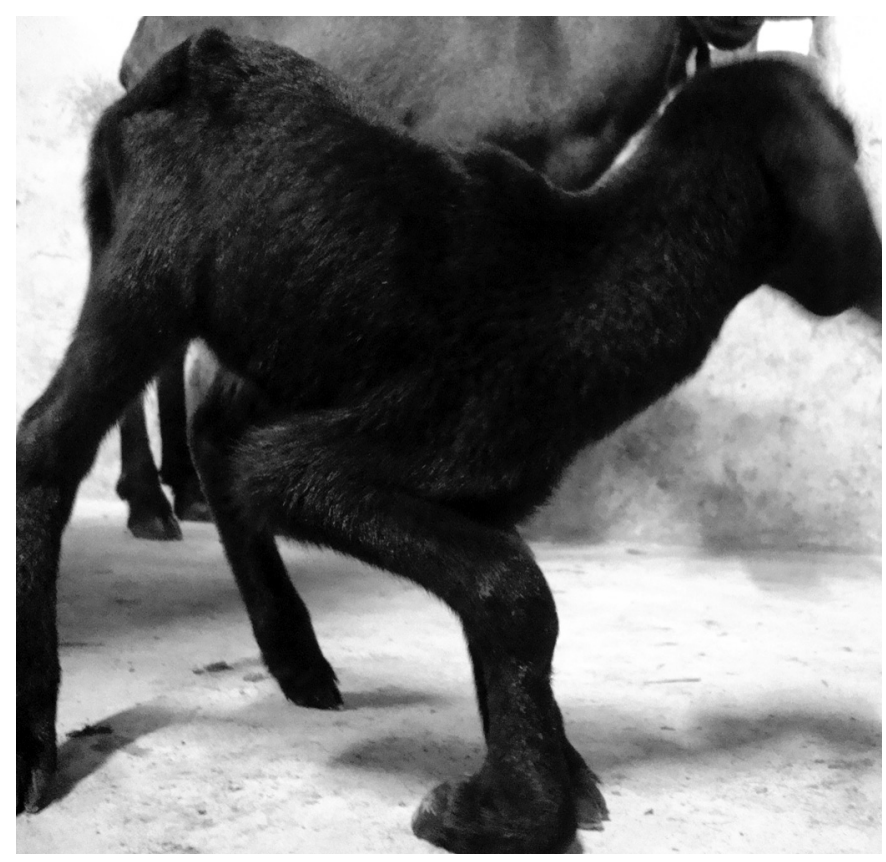

Fig.1. Cordeiro mostrando hiperflexão na articulação inter-falangeana proximal no membro torácico direito, na intoxicação por Mimosa tenuiflora.

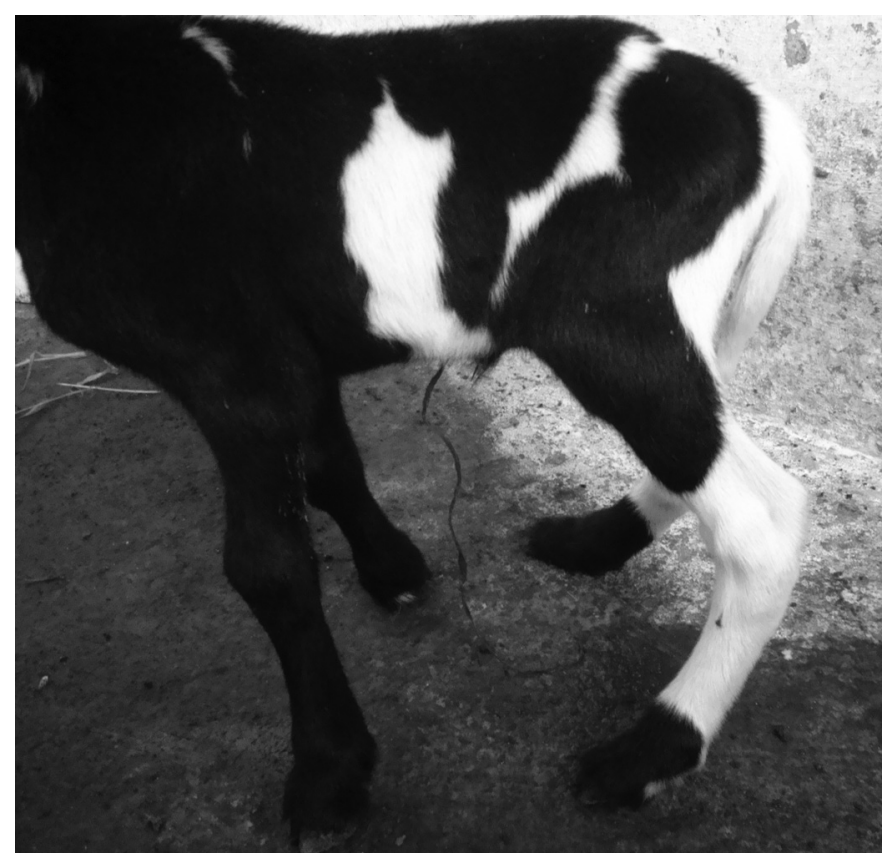

Fig.2. Cordeiro mostrando hiperflexão dos membros pélvicos na região da articulação tarso-metatársica, na intoxicação por Mimosa tenuiflora. tubro de 2009 a janeiro de 2010), quando estavam pastejando na área com $M$. tenuiflora e três não emprenharam durante o período seco, mostrando cio e acasalando repetidamente. Uma ovelha que emprenhou abortou aos 139 dias de gestação e as outras cinco pariram cordeiros sem malformações, quatro partos simples e um gemelar. As três ovelhas que não emprenharam no período seco permaneceram na área experimental com o carneiro, após o início das chuvas, em janeiro de 2010, e pariram cordeiros normais em junho do mesmo ano. As 32 ovelhas do rebanho utilizado como controle pariram 39 cordeiros sem malformações. Uma teve um parto duplo distócico, morrendo os dois cordeiros.

\section{DISCUSSÃO}

Neste trabalho observou-se a ocorrência de malformações em cordeiros nascidos de ovelhas que ingeriram Mimosa tenuiflora, confirmando seu efeito teratogênico, semelhante ao observado em trabalhos anteriores em caprinos (Pimentel et al. 2007) e ratos (Medeiros et al. 2008). Todavia, houve malformações de membros e não foram observadas malformações da face, as quais são relatadas em casos espontâneos associados à ingestão de $M$. tenuiflora (Nóbrega Jr et al. 2005, Dantas et al 2010). Em ovinos, o período de suscetibilidade máxima às malformações começa quando a organogênese é iniciada em torno do $16^{\circ}$ dia de gestação e se estende na maioria dos órgãos até o $34^{\circ}$ dia, quando termina a organogênese e começa a fase fetal (Sinowatz 2010). Malformações ocorrem, também, em outras fases da gestação, quando há alteração na conformação de uma estrutura do corpo que já sofreu diferenciação normal, após o período de organogênese (Radostits et al. 2007). É o caso da artrogripose, que ocorre devido à diminuição dos movimentos fetais (Gardner et al. 1998). Panter et al. (1998) sugerem que esse tipo de malformação poderia ocorrer por ação de bloqueio neuromuscular. As malformações observadas neste experimento regrediram após 14 e 28 dias, respectivamente. Isso pode ser porque a fixação artrogripótica dos membros resultou de mobilidade fetal restrita, mas não o suficiente pra causar uma malformação permanente. A gravidade da lesão depende, provavelmente, do grau de interferência do principio ativo ingerido e da duração da redução dos movimentos fetais. 0 efeito teratogênico é dose-dependente e a incidência, o tipo e a severidade da malformação dependem da composição do principio ativo, do estágio da gestação em que ocorre a ingestão e da quantidade do teratógeno ingerido (Panter et al. 1998, Welch et al. 2011). Alcaloides esteroidais estão associados com malformações causadas por Astragalus lentiginosus, Conium maculatum, Lupinus spp, Nicotiana glauca, Nicotiana tabacum, Oxytropis spp. e Veratrum californicum (Keeler 1990, Cheeke 1998, Gardner et al. 1998, Panter et al. 2000, 2011, James et al. 2004). 0 princípio ativo de M. tenuiflora é, ainda, desconhecido, mas alcaloides derivados da triptamina foram isolados em folhas e sementes (Gardner et al. 2011).

Quando doses altas de princípios ativos ingeridas forem capazes de causar toxicidade materna podem resultar em embrioletalidade ou mortalidade fetal (Panter et al. 1994, Bernardi 2002, Welch et al. 2011). M. tenuiflora 
não tinha sido, ainda, associada com abortos, o que ficou evidenciando neste experimento. Algumas plantas como Astragallus spp. e Oxytropis spp., além de causar deformações podem causar, também, morte fetal e aborto (Panter et al. 1994).

No Grupo 2, três ovelhas retornaram repetidamente ao cio e acasalaram mas não emprenharam, possivelmente devido a mortalidade embrionária, como foi observado em trabalho com caprinos (Dantas et al. 2012). Outras plantas associadas com malformações causam, também, mortalidade e reabsorção embrionária, como Veratrum californicum, quando é ingerida por ovelhas prenhes durante o período de 14 a 21 dias de gestação (Keeler 1990).

M. tenuiflora pode representar um grande obstáculo ao desempenho reprodutivo em ovinos no semiárido; portanto, é importante evitar que ovelhas consumam M. tenuiflora durante o período de acasalamento ou gestação. Numa região onde a disponibilidade de forragem é limitada durante o período de estiagem, a melhor alternativa é a de armazenar forragem na forma de feno e silagem durante o período das chuvas para alimentar as fêmeas prenhes na seca.

Agradecimentos.- Trabalho financiado pelos Institutos Nacionais de Ciência e Tecnologia (INCT) para o Controle das Intoxicações por Plantas (CNPq. Proc. 573534/2008-0).

\section{REFERÊNCIAS}

Bernardi M.M. 2002. Exposição aos medicamentos durante o período perinatal, p.692-699. In: Spinosa H.S., Gorniak S.L. \& Bernardi M.M. (Eds), Farmacologia Aplicada à Medicina Veterinaria. $3^{\underline{a}}$ ed. Guanabara Koogan, Rio de Janeiro, RJ. 752p.

Cheeke P.R. 1998. Natural Toxicants in Feeds, Forages, and Poisonous Plants. $2^{\text {nd }}$ ed. Danville, Interstate Publishers, Danville, p.691-699.

Dantas A.F.M., Riet-Correa F., Medeiros R.M.T., Lopes J.R., Gardner D.R., Panter K. \& Mota R.A. 2012. Embryonic death in goats caused by the ingestion of Mimosa tenuiflora. Toxicon 59 (5) 555-557.

Dantas A.F.M., Riet-Correa F., Medeiros R.M.T., Galiza G.J.N., Pimentel L.A., Anjos B.L. \& Mota R.A. 2010. Malformações congênitas em ruminantes no semiárido do Nordeste Brasileiro. Pesq. Vet. Bras. 30(10):807-815.

Gardner D.R., Panter K.E., Stegelmeier B.L., James L.F., Ralphs M.H., Pfister J.A. \& Schoch T.K. 1998. Livestock poisoning by teratogenic and hepatotoxic range plants, p.303-306. In: Garland T. \& Barr A.C. (Eds), Toxic Plants and Other Natural Toxicants. CAB International, Wallingford, UK. $585 p$.

Gardner D.R., Riet-Correa F. \& Panter K.E. 2011. Alkaloid profiles of Mimosa tenuiflora and associated methods of analysis, p.600-605. In: Riet Cor- rea F., Pfister J., Schild A.L. \& Wierenga T. (Eds), Poisoning by Plants, Mycotoxins and Related toxins. CAB International, Wallingford, UK. 739p.

James L.F., Panter K.E., Gaffield W. \& Molyneux R.J. 2004. Reviews: Biomedical applications of poisonous plant research. J. Agric. Food Chem. 52:3211-3230.

Keeler R.F. 1984. Teratogens in plants. J. Anim. Sci. 58:1029-1039.

Keeler R.F. 1990. Early embryonic death in lambs induced by Veratrum californicum. Cornell Vet. 80:203-207.

Medeiros J.M., Tabosa I.M., Simões S.V.D., Nóbrega Júnior J.E., Vasconcelos J.S. \& Riet-Correa F. 2005. Mortalidade perinatal em caprinos no semi-árido da Paraíba. Pesq. Vet. Bras. 25(4):201-206.

Medeiros R.M.T., Figueiredo A.P.M., Benício T.M.A., Dantas F.P.M. \& Riet-Correa F. 2008. Teratogenicity of Mimosa tenuiflora seeds to pregnant rats. Toxicon 51:316-319.

Nóbrega Júnior J.E., Riet-Correa F., Nóbrega R.S., Medeiros J.M., Vasconcelos J.S., Simões S.V.D. \& Tabosa I.M. 2005. Mortalidade perinatal de cordeiros no semi-árido da Paraíba. Pesq. Vet. Bras. 25(3):171-178.

Panter K.E., James L.F., Gardner D.R. \& Molyneux R.J. 1994. The effects of poisonous plants on embryonic and fetal development in livestock, p.325-332. In: Colegate S.M. \& Dorling P.R. (Eds), Plant Associated Toxins. CAB International, Wallingford, UK. 581p.

Panter K.E., Gardner D.R., Shea R.E., Molyneux R.J. \& James LF. 1998. Toxic and teratogenic piperidine alkaloids from Lupinus, Conium and Nicotia$n a$ species, p.345-350. In: Garland T. \& Barr A.C. (Eds), Toxic Plants and other Natural Toxicants. CAB International, Wallingford, UK. 585p.

Panter K.E., Weinzweig J., Gardner D.R., Stegelmeier B.L. \& James L.F. 2000. Comparison of cleft palate induction by Nicotiana glauca in goats and sheep. Teratology 61:203-210.

Panter K.E., Wlech K.D., Lee S.T., Gardner D.R., Steegelmeier B.L., Ralphs M.H., Davis T.Z., Green B.T., Pfister J.A. \& Cook D. 2011. Plants teratogenic to livestock in the United States, p.236-242. In: Riet Correa F., Pfister J., Schild A.L. \& Wierenga T. (Eds), Poisoning by Plants, Mycotoxins and Related toxins. CAB International, Wallingford, UK.. 739p.

Pimentel L.A., Riet Correa F., Gardner D., Panter K.E., Dantas A.F.M., Medeiros R.M.T., Mota R.A. \& Araújo J.A.S. 2007. Mimosa tenuiflora as a cause of malformations in ruminants in the northeastern Brazilian semiarid rangelands. Vet. Pathol. 44(6):928-931.

Radostits O.M., Gay C.C., Hinchcliff K.W. \& Constable P.D. 2007. Disease of the newborn, p.127-172. In: Radostits O.M., Gay C.C., Hinchcliff K.W. \& Constable P.D. (Eds), Veterinary Medicine: A textbook of the diseases of cattle, horses, sheep, pigs and goats. $10^{\text {th }}$ ed. Saunders Elsevier, Philadelphia. 2162p.

Sinowatz F. 2010. Teratology, p.339-382. In: Hyttel P., Sinowatz F. \& Vejlsted M. (Eds), Essential of Domestic Animal Embriology. Elsevier, China. 453p.

Welch K.D., Lee S.T., Gardner D.R., Panter K.E., Steegelmeier B.L. \& Cook D. 2011. Dose-response evaluation of Veratrum californicum in sheep, p.243-250. In: Riet Correa F., Pfister J., Schild A.L. \& Wierenga T. (Eds), Poisoning by Plants, Mycotoxins and related Toxins. CAB International, Wallingford, UK. 739p. 